\title{
Assessment of Flex-Grid/MCF Optical Networks with ROADM Limited Core Switching Capability
}

\author{
R. Rumipamba-Zambrano ${ }^{1}$, J. Moreno ${ }^{2}$, P. Pavón-Marino ${ }^{2}$, J. Perelló ${ }^{1}$, S. Spadaro ${ }^{1}$ and J. Solé-Pareta ${ }^{1}$ \\ ${ }^{1}$ Universitat Politècnica de Catalunya (UPC), Jordi Girona 1-3, 08034 Barcelona, Spain \\ ${ }^{2}$ Universidad Politécnica de Cartagena (UPCT), Cuartel de Antigones, Plaza del Hospital 1, 30202 Cartagena, Spain \\ E-mail: rrumipam@ac.upc.edu, \{javier.moreno,pablo.pavon\}@upct.es
}

\begin{abstract}
The majority of the research studies on Flex-Grid over multi-core fiber (Flex-Grid/MCF) networks are built on the assumption of fully non-blocking ROADMs (FNB-ROADMs), able to switch any portion of the spectrum from any input core of any input fiber to any output core of any output fiber. Such flexibility comes at an enormous extra hardware cost. In this paper, we explore the trade-off of using ROADMs that impose the so-called core continuity constraint (CCC). Namely, a CCC-ROADM can switch spectrum from a core on an input fiber to a chosen output fiber, but cannot choose the specific output core. For instance, if all fibers have the same number of cores, the $i$-th core in the input fibers can be just switched to the $i$-th core in the output fibers. To evaluate the performance vs. cost trade-off of using CCCROADMs, we present two Integer Linear Programming (ILP) formulations for optimally allocating incoming demands in FlexGrid/MCF networks, where the CCC constraint is imposed or not, respectively. A set of results are extracted applying both schemes in two different backbone networks. Transmission reach estimations are conducted accounting for the fiber's linear and non-linear effects, as well as the inter-core crosstalk (ICXT) impairment introduced by laboratory MCF prototypes of 7, 12 and 19 cores. Our numerical evaluations show that the performance penalty of CCC is minimal, i.e., below $1 \%$ for 7 and 12-core MCF and up to $10 \%$ for 19 -core MCF, while the cost reduction is large. In addition, results reveal that the ICXT effect can be significant when the number of cores per MCF is high, up to a point that equipping the network with 12-core MCFs can yield superior effective capacity than with 19-core MCFs.
\end{abstract}

Keywords-Space Division Multiplexing; Flex-Grid; Multi-Core Fiber; inter-core Cross-Talk; ROADM; core switching.

\section{INTRODUCTION}

Space Division Multiplexing (SDM) technology has become the "next-frontier" of fiber optics to scale up the capacity of current optical fiber systems [1]. Different SDM technological solutions have appeared to date. The basic one consists in upgrading currently deployed telecom operators' networks to convert them into Multi-Fiber (MF) infrastructures, where every link bundles several single-mode fibers. But as with Wavelength-Division Multiplexing (WDM), parallelization is a must for SDM to become economically attractive, hence novel fiber designs are required [2]. A technological solution to achieve this goal can rely on a single fiber core, large enough to transmit multiple guided modes, thus having a Multi-Mode Fiber (MMF). If these transmitted modes are limited to only a few, such fiber technologies are called Few-Mode Fibers (FMFs). Another possibility to realize SDM is to employ fibers with several single-mode cores, called Multi-Core Fibers (MCFs). If these cores carry a few modes each, such fibers are referred to as Few-Mode Multi-Core Fibers (FM-MCFs).

These novel fibers introduce a new impairment that should be taken into account. Indeed, the coupling between modes or cores has to be considered in order to determine if equalization based on end-to-end Multiple-Input and Multiple-Output (MIMO) processing is necessary. In particular, MCFs with several singlemode cores are attractive, given their extremely low inter-core crosstalk (ICXT) values [3]-[5], alleviating the need for MIMO equalization. To further reduce ICXT, heterogeneous MCFs (cores with different characteristics) have also been designed, which may be required in long-haul communications for core counts larger than 30 [6].

Over the last years, Flex-Grid has appeared to replace traditional WDM, given its superior flexibility to make the most of the available fiber spectral resources. Such is the case that its standardization is ongoing within the ITU-T [7]. Flex-Grid technology enables transmissions at ultra-high bit-rates by concatenating multiple adjacent flexible sub-channels, thus forming a super-channel. This is supported by Software Defined Optical Bandwidth Variable Transponders (BVTXPs). In a Flex-Grid/MCF network scenario like the one considered in this work, MCFs open another degree of flexibility for super-channel configuration, namely, the spatial domain.

Reconfigurable Optical Add-Drop Multiplexer (ROADM) devices are responsible to automatically add, drop, and bypass lightpaths at network nodes. Several ROADM architectures have been proposed to offer switching flexibility to next generation Elastic Optical Networks [8]. The two basic ones are the so-called Broadcast-and-Select (B\&S) and Route-and-Select $(\mathrm{R} \& \mathrm{~S})$. The $\mathrm{B} \& \mathrm{~S}$ architecture yields reduced cost, power consumption and optical/electronic complexity, as well as low overall system penalties. On the other hand, the R\&S roughly doubles the number of Spectrum Selective Switches (SSSs) to provide superior isolation on the blocking ports and a constant insertion loss regardless of the port count [9]. According to [9], the B\&S architecture seems a good choice for ROADMs with node degree $(N)$ lower or equal than 9 , while for $N>9, \mathrm{R} \& \mathrm{~S}$

(C) 2017 IEEE. Personal use of this material is permitted. Permission from IEEE must be obtained for all other uses, in any current or future media, including reprinting/republishing this material for advertising or promotional purposes,creating new collective works, for resale or redistribution to servers or lists, or reuse of any copyrighted component of this work in other works. 
benefits compensate its extra cost.

Having a full interconnection at the ROADM between ports and routes may require an enormous node complexity. In this regard, multiple studies exist in the literature evaluating the performance penalty of ROADM architectures where some internal blocking is allowed. For example, the internal blocking for Flex-Grid optical networks is evaluated in [10].

With the new space dimension enabled by SDM, new performance vs. cost trade-offs appear. Initial works in the literature present allocation schemes for MCF networks assuming fully non-blocking ROADMs ([13] [14]). However, the complexity of the MCF-ROADM architectures greatly increases if a non-blocking ROADM is pursued. In particular, when directly applying $B \& S$ or $R \& S$ concepts, if each input/output fiber supports $S$ spatial channels, then (i) the number of required SSS devices at ROADMs must be multiplied by $S$, and (ii) its individual size is also multiplied.

To simplify these architectures, alternative B\&S and R\&S solutions have been proposed in [11] and [12]. The so-called Architecture on Demand [11] can avoid underutilized hardware modules by sharing them on demand via node programmability capabilities. And Joint-switching [12] allows reducing the number of SSS devices by switching one spectral slice in all cores at once (i.e., a spatial super-channel), in exchange of increasing the port count of these SSS devices.

The work in this paper is an attempt in this line based on a different approach: the core continuity constraint (CCC). We firstly propose alternative ROADM architectures that trade core switching flexibility for superior cost-efficiency. In particular, in addition to the spectrum continuity and spectrum contiguity constraints imposed by Flex-Grid optical networks, the CCC will have to be considered by assigning spectral slices to traffic demands in the same core index in all MCF links along the endto-end path. As a next step, we assess their performance. For this, we present two Integer Linear Programming (ILP)-based allocation schemes that are able to optimally choose lightpath routes and spectrum in MCF networks based on (i) ROADMs with the CCC (referred to as CCC-ROADM), (ii) fully nonblocking ROADMs (referred to as FNB-ROADM, i.e. not imposing the CCC).

The remainder of this paper is structured as follows. Section II describes the transmission reach estimation model for MCFenabled optical networks used to obtain the numerical results. Section III elaborates on the analysis of the internal blocking in the Flex-Grid/SDM ROADMs. Section IV presents the ILP formulations for the resource allocation problem in FlexGrid/MCF optical networks in FNB-ROADM (subsection IV.A) and CCC-ROADM (subsection IV.B) architectures. Section V presents the obtained numerical results. Finally, section VI draws up the main conclusions of this paper and envisions future research lines.

\section{TRANSMisSiON REACH ESTIMATION}

An accurate estimation of the maximum Transmission Reach (TR) of the optical signals across the underlying (e.g., Flex-
Grid/MCF) transparent optical network infrastructure becomes paramount in any connection allocation strategy. Otherwise, one can encounter unfeasible communications. However, this is a difficult task, as it depends on many different variables like the type of fiber, the amplification scheme, the dispersion map, the nonlinear effects compensation capability, the modulation format, the channel spacing, and so on.

Recently, the Gaussian noise (GN)-model has been presented in [15], and its reliability for nonlinear fiber propagation has been proven in most cases of interest. An enhanced version (EGN-model) has been also developed to provide even better accuracy in [16], being the previous one an upper bound of the nonlinear interference (NLI) calculation, which leads to a slightly pessimistic reach estimation. In this paper, we assume the same scenario as "Link 1" in [15], whose details are listed as follows:

- $85-\mathrm{km}$ spans of standard single-mode fiber (SSMF).

- Erbium-doped fiber amplifier (EDFA) with noise factor $\mathrm{NF}=5 \mathrm{~dB}$.

- Polarization-multiplexed (PM) quadrature amplitude modulation (PM-QAM).

- Ideal Nyquist WDM (i.e., channel spacing equal to the symbol rate).

- Full Digital Signal Processing (DSP)-based chromatic dispersion compensation.

- No fiber nonlinearities compensation.

- No Polarization-Mode Dispersion (PMD).

- State-of-the-art soft Forward Error Correction (FEC) with $20 \%$ overhead at pre-FEC BER of $2.7 \times 10^{-2}$.

- 3-dB Signal-to-Noise Ratio (SNR) margin from ideal performance and 1.5-dB penalty of soft FEC with respect to infinite-length codes ideal performance.

The estimated transmission reach corresponding to each modulation format is provided in Table I. This is a pessimistic estimation for several reasons: (1) as previously mentioned, the GN-model is intrinsically pessimistic, (2) the channel spacing is set to its theoretical minimum, while in our simulations guardbands (GBs) are included between adjacent channels, (3) no fiber nonlinearities compensation is assumed and (4) no Raman amplification is employed.

TABLE I.
\begin{tabular}{|c|c|c|c|}
\hline BPSK & QPSK IN KM GIVEN BY THE GN-MODEL $[15]$ \\
\hline$>20000$ & 9000 & 16-QAM & 64-QAM \\
\hline
\end{tabular}

TABLE II. ICXT-LIMITED TR IN KM FOR DIFFERENT MCF

\begin{tabular}{|c|c|c|c|c|}
\hline $\boldsymbol{C}$ & BPSK & QPSK & 16-QAM & 64-QAM \\
\hline $\mathbf{7}[\mathbf{3}]$ & 4683271 & 2347195 & 589589 & 148098 \\
\hline $\mathbf{1 2}[\mathbf{4}]$ & 24322 & 12190 & 3062 & 769 \\
\hline $\mathbf{1 9}[\mathbf{5}]$ & 4755 & 2383 & 599 & 150 \\
\hline
\end{tabular}

TABLE III. OVERALL TR ESTIMATION IN KM

\begin{tabular}{|c|c|c|c|c|}
\hline $\boldsymbol{C}$ & BPSK & QPSK & 16-QAM & 64-QAM \\
\hline $\mathbf{7}[\mathbf{3}]$ & $>20000$ & 9000 & 2000 & 600 \\
\hline $\mathbf{1 2}[\mathbf{4}]$ & $>20000$ & 9000 & 2000 & 600 \\
\hline $\mathbf{1 9}[\mathbf{5}]$ & 4755 & 2383 & 599 & 150 \\
\hline
\end{tabular}

To estimate the transmission reach in a MCF scenario, ICXT 
has to be taken into account. A simple way to do it is by considering the worst ICXT introduced by the fiber along propagation and the maximum linear in-band XT tolerated by each modulation format for a $1 \mathrm{~dB}$ Optical Signal-to-Noise Ratio (OSNR) penalty [17]. Assuming the homogeneous 7-core $(C=7)$, 12-core $(C=12)$, 19-core $(C=19)$ MCF prototypes presented in [3]-[5] the measured ICXT is $-84.7 \mathrm{~dB} / \mathrm{km},-61.9$ $\mathrm{dB} / \mathrm{km}$ and $-54.8 \mathrm{~dB} / \mathrm{km}$ (referred to $1550 \mathrm{~nm}$ ), respectively. The maximum reach obtained for each MCF is shown in Table II, where a 4- $\mathrm{dB}$ margin has been considered similarly to the nonlinear propagation case. Recently, a more sophisticated method of estimating the impact of ICXT based on its random nature has been proposed [18]. It has been shown, however, that estimations based on average ICXT may be too optimistic. Further investigation is thus required to validate the model.

As can be seen in Table I and II, TR is limited by the GNmodel for both 7-core and 12-core MCF and by ICXT for 19core MCF. This is reflected in the overall TR estimations shown in Table III, which are the ones used later on in Section V to obtain the numerical results.

\section{FLEX-GRID/SDM ROADM INTERNAL BLOCKING ANALYSIS}

As previously mentioned, ROADMs are in charge of automatically adding, dropping or bypassing lightpaths in FlexGrid Optical Networks. Commercial ROADMs commonly use the broadcast-and-select (B\&S) scheme to switch traffic. The incoming optical signals from adjacent nodes are broadcasted to all available directions' modules within the ROADM by a splitter (power-loss-passive device). At the output ports, signals from all input fibers enter an SSS, the Flex-Grid version of the WSS (Wavelength Selective Switch) device.

SSSs are able to switch any frequency slot (hereinafter only referred to as slot) at any of its input ports, to any of its outputs ports, and vice-versa [19]. They are the most expensive elements in a ROADM design. As mentioned, in B\&S architectures, one SSS is located per output fiber (and drop modules, potentially). Each SSS should have as many input ports as input fibers (and add modules, potentially). In R\&S architectures, SSSs are also located per input fiber, so that the input signal is not broadcasted to all output ports, but switched to the desired output fiber. Thanks to that, isolation is increased and signal attenuation caused by the broadcast operation is eliminated.

In MCF-enabled networks, ROADMs must evolve to manage the traffic among all available cores. Fig. 1 shows the architecture of B\&S FNB-ROADM with $F$ input/output fibers, each with $C$ cores. Observing the architecture we see that:

- The number of splitters and of SSSs is FxC and grows linearly with the number of cores.

- The number of input ports of each SSS is FxC and grows linearly with the number of cores.

- The attenuation caused by the splitters is approximately $10 \log (F \times C)$, thus growing logarithmically with the number of cores.

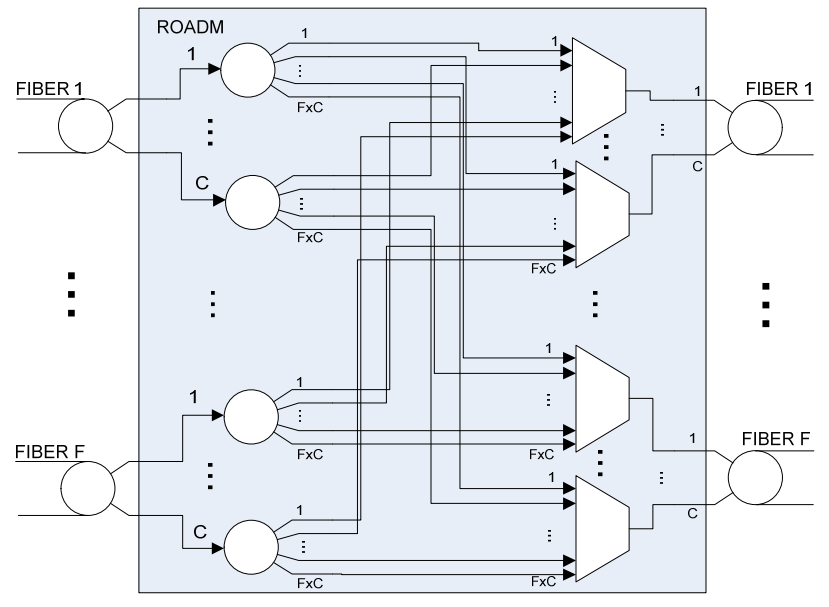

Fig. 1. B\&S FNB node architecture

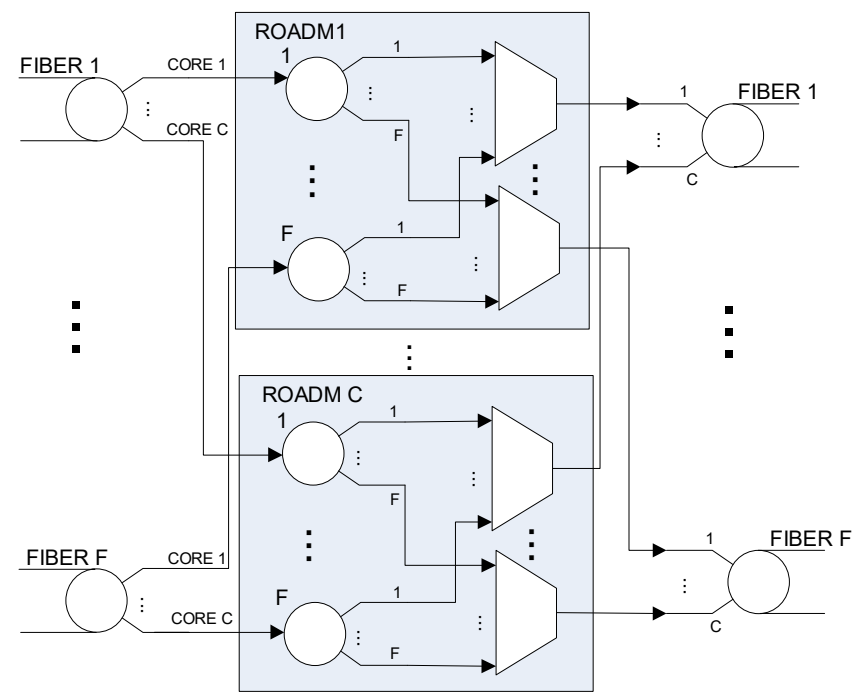

Fig. 2. B\&S CCC node architecture

Fig. 2 shows the scheme of a B\&S MCF-ROADM subject to the $C C C$, for a node of degree $F$ and $C$ cores per fiber. Note that it can be built as a parallel arrangement of $C$ regular ROADMs of degree F. Looking at the architecture we see that:

- As in the fully non-blocking case, the number of splitters and of SSSs is FxC and grows linearly with the number of cores.

- The number of input ports of each SSS is now $F$ instead of FxC, and does not grow with the number of cores.

- The attenuation (in $\mathrm{dB}$ ) caused by the splitters is approximately $10 \log (F)$ instead of $10 \log (F \times C)$, and does not grow with the number of cores.

TABLE IV. SPLITTER ATENUATION IN $[d B]$ (CCC, FNB)

\begin{tabular}{|c|c|c|c|c|c|c|}
\hline $\boldsymbol{C}$ & \multicolumn{2}{|c|}{$\boldsymbol{F}=\mathbf{2}$} & \multicolumn{2}{c|}{$\boldsymbol{F}=\mathbf{4}$} & \multicolumn{2}{c|}{$\boldsymbol{F}=\mathbf{8}$} \\
\hline & CCC & FNB & CCC & FNB & CCC & FNB \\
\hline $\mathbf{7}$ & 3.01 & 11.46 & 6.02 & 14.47 & 9.03 & 17.48 \\
\hline $\mathbf{1 2}$ & 3.01 & 13.80 & 6.02 & 16.81 & 9.03 & 19.82 \\
\hline $\mathbf{1 9}$ & 3.01 & 15.80 & 6.02 & 18.81 & 9.03 & 21.82 \\
\hline
\end{tabular}


TABLE V. NuMBER OF REQUIRED INPUT PORTS PER SSS (CCC, FNB)

\begin{tabular}{|c|c|c|c|c|c|c|}
\hline $\boldsymbol{C}$ & \multicolumn{2}{|c|}{$\boldsymbol{F}=\mathbf{2}$} & \multicolumn{2}{c|}{$\boldsymbol{F}=\mathbf{4}$} & \multicolumn{2}{c|}{$\boldsymbol{F}=\mathbf{8}$} \\
\hline & CCC & FNB & CCC & FNB & CCC & FNB \\
\hline $\mathbf{7}$ & 2 & 14 & 4 & 28 & 8 & 56 \\
\hline $\mathbf{1 2}$ & 2 & 24 & 4 & 48 & 8 & 96 \\
\hline $\mathbf{1 9}$ & 2 & 38 & 4 & 76 & 8 & 152 \\
\hline
\end{tabular}

Table IV and Table V compare the complexity of the CCC and FNB MCF-ROADMs. Results are compiled for node degrees $F=\{2,4,8\}$ and number of cores per fiber $C=\{7,12,19\}$. Table IV plots the minimum signal attenuation in the broadcast stage of the ROADM when an ideal splitter is used. We see that FNB attenuations steadily grow with the number of cores, while the attenuation is insensitive to the number of cores in CCCROADMs. Note that higher attenuations result in more expensive and noisy internal optical amplifiers needed. As a comparison, a typical EDFA is able to amplify in the order of 20-25 dB.

Table $\mathrm{V}$ compares the number of input ports required in the SSS of both CCC- and FNB-ROADMs. In both cases, we assume that the ROADMs have no add/drop modules for adding and dropping lightpaths at the node. For each add/drop module, the number of input ports would be increased by one. We shadow the cells where the number of input or output ports of the SSS exceeds 32. This number is comparable to the largest state-of-the-art commercial SSS in [20]. Results reveal the excellent scalability properties of the CCC-ROADMs, and highlight the technical difficulties in building medium or largescale non-blocking MCF-ROADMs.

\section{ILP FORMULATION}

In this section, we present the ILP formulation for the routing, modulation, core and spectrum assignment (RMCSA) problem with the CCC- and FNB-ROADM architectures mentioned in Section III. To this end, the following common input parameters are introduced:

- $\mathcal{N}$ : Set of Nodes.

- $\varepsilon$ : Set of MCF fibers.

- $\mathcal{C}$ : Set of cores per MCF, so that $|\mathcal{C}|=C$

- $\mathcal{D}$ : Set of offered IP traffic demands.

- $\delta$ : Set of available slots per core.

- $\mathcal{T}$ : Set of transponder types available in the network. For each transponder type $t \in \mathcal{T}, r(t)$ is the line rate of the transponder, and $s(t)$ is the number of slots occupied by the signal of this transponder.

- $h_{d}, d \in \mathcal{D}$ : Offered traffic per IP demand, measured in Gbps, which can be carried by one or several lightpaths.

- $\mathcal{P}_{d t}, d \in \mathcal{D}, t \in \mathcal{T}$ : For each demand and transponder type, list of admissible paths. In our case, the $k$ loop-less shortest paths (in $\mathrm{km}$ ) between the demand end nodes, excluding those outside transmission reach. These paths are potential routes to carry the traffic of the demand with a transponder of the given type $t$.

- $\mathcal{P}$ : Set of all admissible paths in the network $(\mathcal{P}=$ $\left.\cup_{d \in \mathcal{D}, t \in \mathcal{T}} \mathcal{P}_{d t}\right)$. We denote as $\mathcal{P}_{e}$ those paths traversing
MCF $e$. Given a path $p, d(p)$ denotes the amount of IP demand it is associated to, $l(p)$ denotes the number of hops it is associated to (i.e., the number of MCF links $e$ that traverse the path $p$ between the end nodes), and $t(p)$ denotes the transponder it is associated to. For simplicity, we denote as $r_{p}=r(t(p))$ the rate of $p$, and $s_{p}=$ $s(t(p))$ the number of slots occupied.

- $\delta\left(p, s_{o}\right)$ is the set of slots occupied by path $p$, when its initial slot is $s_{o}$. This means, starting in $s_{o}$, as many contiguous slots as $s_{p}$.

\section{A. ILP formulation for RMCSA with FNB-ROADM}

In this subsection we present the details of the proposed ILP formulation for RMCSA problem with fully non-blocking ROADM architecture, where we introduce the following decision variable:

$x_{p s}, p \in \mathcal{P}, s \in \mathcal{S}$ : binary decision variable; 1 if path $p$ has a first slot $s$ (the $s(t(p))$ slots occupied are contiguous); 0 otherwise.

$$
\min \sum_{p \in \mathcal{P}, s \in \mathcal{S}} s_{p} l(p) x_{p s}
$$

subject to:

$$
\begin{gathered}
\sum_{p \in \mathcal{P}_{d}, s \in \mathcal{S}} r_{p} x_{p s} \geq h_{d}, \forall d \in \mathcal{D} \\
\sum_{p \in \mathcal{P}_{e}, s_{o}: s \in \mathcal{S}\left(p, s_{o}\right)} x_{p s_{o}} \leq C, \forall e \in \mathcal{E}, s \in \mathcal{S}
\end{gathered}
$$

Objective function (1a) minimizes the total number of slots used in the network. Constraint (1b) represents the fulfillment of the offered traffic for each IP demand: the sum of the operational line rates is at least the offered IP traffic. Finally, constraint (1c) means the slot-clashing constraint: one slot $s$ of one fiber $e$ can be used by $C$ lightpaths (one per core).

\section{B. ILP formulation for RMCSA with CCC-ROADM}

In this subsection we present the details of the proposed ILP formulation for RMCSA problem using ROADM with the core continuity constraint, where we introduce the following decision variable:

$x_{p c s}, p \in \mathcal{P}, c \in \mathcal{C}, s \in \mathcal{S}$ : binary decision variable; 1 if path $p$ uses the core index $c$ and it has a first slot $s$ (the $s(t(p)$ ) slots occupied are contiguous); 0 otherwise.

$$
\min \sum_{p \in \mathcal{P}, c \in \mathcal{C}, s \in \mathcal{S}} s_{p} l(p) x_{p c s}
$$

subject to:

$$
\begin{gathered}
\sum_{p \in \mathcal{P}_{d}, c \in C, s \in \mathcal{S}} r_{p} x_{p c s} \geq h_{d}, \forall d \in \mathcal{D} \\
\sum_{p \in \mathcal{P}_{e}, s_{o}: s \in \mathcal{S}\left(p, s_{o}\right)} x_{p c s_{0}} \leq 1, \forall e \in \mathcal{E}, c \in \mathcal{C}, s \in \mathcal{S}
\end{gathered}
$$

Similarly to ILP formulation (1), objective function (2a) minimizes the total number of slots that are used in the network. Constraint (2b) represents the fulfillment of the offered traffic for each demand. Finally, constraint (2c) means the slot-clashing and core continuity constraints: one slot $s$ and core index $c$ cannot be used by two lightpaths. 

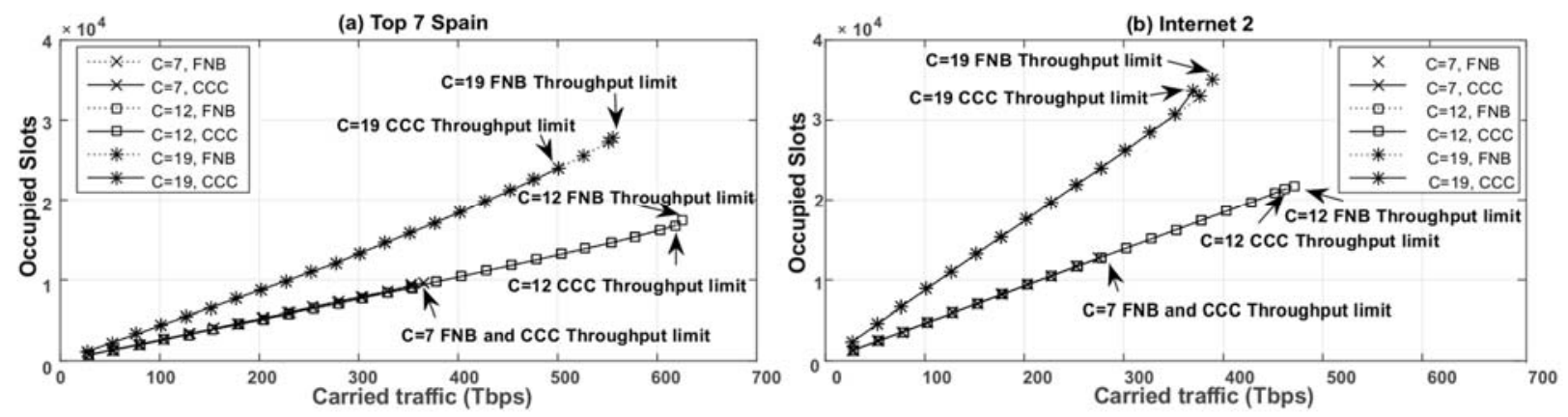

Fig. 3. Occupied slots vs. carried traffic in: (a) T7S and (b) I2 networks.

\section{NUMERICAL RESULTS}

This section collects and analyzes extensive results obtained for evaluating the proposed CCC-ROADM architecture in FlexGrid/MCF optical networks using the ILP formulations presented in Section IV. These formulations have been implemented in the Net2Plan tool [21] [22], which interfaces with the CPLEX solver through the JOM library [23].

The simulation was run on a server with a 16-core CPU and 32 GB RAM. The JOM library was configured so that in each algorithm run, if the ILP did not end after one hour of execution, the optimum solution found so far was returned.

Two reference topologies have been considered: the Top 7 Spain (T7S) with an average link length of $272.9 \mathrm{~km}$ and 2.29 node degree; and the Internet2 (I2) with an average link length of $1062.6 \mathrm{~km}$ and 2.89 node degree. These topologies are available in [23]. Each network link represents a $C$-core $\mathrm{MCF}$ $(C=\{7,12,19\})[3]-[5]$ with an available spectrum per core of 120 slots of $12.5 \mathrm{GHz}$ spectral width.

Incoming IP traffic demands considered are generated by scaling a seminal traffic matrix. Such a seminal matrix is created by sampling a uniform random $(0,1)$ distribution for each node pair. The lightpaths satisfying the IP traffic demands can use any transponder $t \in \mathcal{T}$ at any line rate $r(t)$ belonging to set $\mathrm{R}=\{40,100,400\} \mathrm{Gb} / \mathrm{s}$. The line rates are obtained using flexible symbol rate and different modulation formats (BPSK, QPSK, 16-QAM, 64-QAM) according to the transmission distance requirements. For this, the most efficient modulation format is selected according to the TR estimations shown in Table III. Each transponder occupies a certain number of slots

$s(t)$ which includes a $10 \mathrm{GHz}$ GB [14] and it reads $\left\lceil\frac{\text { line_rate } / S E+G B}{W}\right\rceil$, where $S E$ is the spectral efficiency of the selected modulation format and $W$ the spectral width occupied by one slot. Note that ILPs are able to optimally choose those transponders to use, and that different lightpaths, even supporting the same IP demand, can use different transponders at different rates (i.e., experiencing different TRs). In the path list elaboration we enumerated $k=5$ shortest paths in $\mathrm{km}$ for each transponder type (filtering out those outside TR).

Fig. 3(a) and Fig. 3(b) display the number of slots occupied in the network for increasing IP traffic loads, assuming that either CCC- or FNB-ROADM architectures are employed in the two reference topologies. The results show that, the slot occupation in the network is the same or very similar for $C=7$, 12 and 19 for different traffic loads. In these figures, we also observe that the throughput limit (network capacity, i.e., the largest scaled version of the matrix for which an allocation was possible) for $C=19$ with FNB-ROADM architecture is higher than CCC-ROADM one, because the restriction of core switching is more significant as the core count increases.

To extend the analysis of throughput limit, Fig. 4(a) and Fig. 4(b) show it separately for the different ROADM architectures. We see that, the reduction in network capacity when using CCC-ROADM is inexistent for $C=7$, around $1 \%$ for $C=12$ and between $10 \%$ and $5 \%$ for $C=19$ (in T7S and $\mathrm{I} 2$ networks, respectively).

It is worth observing in Figs. 3 and 4 that upgrading the network from $C=7$ to $C=12$ cores results in an appropriate linear increase in the network capacity because of the extra cores.
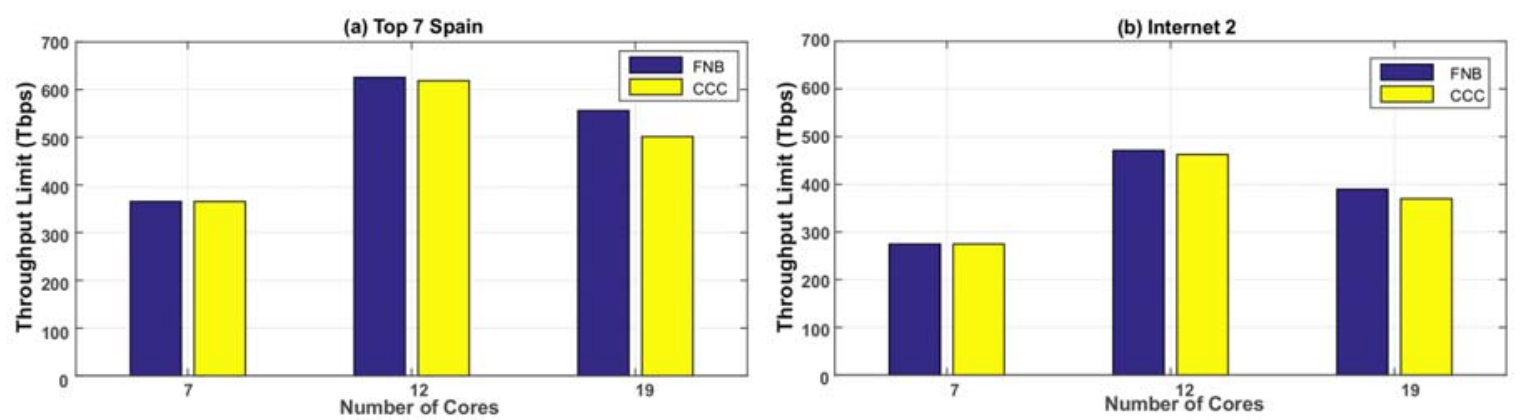

Fig. 4. Throughput limit vs. Number of cores per fiber in: (a) T7S and (b) I2 networks. 
However, if we upgrade again to $C=19$, the maximum network throughput actually decreases. The reason of such behavior is the drastic reduction in the TR caused by ICXT impairment, as observed in Table III. To witness this effect, Table VI collects the network economic efficiency, measured as the ratio between the network throughput (as a measure of the revenues) and the total number of cores in the network (as a measure of the cost). As can be seen, in the $C=19$ case the efficiency drops significantly.

TABLE VI.
\begin{tabular}{|c|c|c|c|}
\hline Topology & $\boldsymbol{C}=\mathbf{7}$ & $\boldsymbol{C}=\mathbf{1 2}$ & $\boldsymbol{C}=\mathbf{1 9}$ \\
\hline T7S & 3.3 & 3.3 & 1.5 \\
\hline $\mathbf{I 2}$ & 1.5 & 1.5 & 0.8 \\
\hline
\end{tabular}

\section{CONCLUSIONS}

In this paper, we evaluate the application of the so-called core continuity constraint (CCC) in Flex-Grid/MCF networks. First, we study the cost benefits associated, in terms of reduction of ROADM complexity. The analysis for B\&S ROADMs show that CCC designs are a significantly cheaper and more scalable form of building ROADMs. For the performance side, we propose two ILPs to allocate resources to lightpaths with CCCand FNB-ROADM architectures. Then, we applied these schemes in exhaustive tests for two reference topologies, and realistic transmission reach calculations for the transponders, considering ICXT, in fibers of 7, 12 and 19 cores. Our tests show an often null, and sometimes minimal performance penalty, caused by internal ROADM blocking when CCC is applied. Also, results discourage the utilization of MCF with 19-cores because the transmission impairments greatly limit the network capacity.

\section{ACKNOWLEDGMENT}

This work has been partially funded by the Spanish national projects SUNSET (TEC2014-59583-C2-1-R), which receives funding from FEDER, Elastic Networks (TEC2015-71932REDT), and ONOFRE (TEC2014-53071-C3-1-P). Rubén Rumipamba is recipient of a full scholarship from Secretaría Nacional de Ciencia y Tecnología (SENESCYT) - Ecuador.

\section{REFERENCES}

[1] P. J. Winzer, "Spatial Multiplexing: The Next Frontier in Network Capacity Scaling," Eur. Conf. Exhib. Opt. Commun., p. We.1.D.1, 2013.

[2] D. J. Richardson, J. M. Fini, and L. E. Nelson, "Space Division Multiplexing in Optical Fibres," Nat. Photonics, vol. 7, pp. 354-362, 2013.

[3] J. Sakaguchi, Y. Awaji, N. Wada, A. Kanno, T. Kawanishi, T. Hayashi, T. Taru, T. Kobayashi, and M. Watanabe, "Space division multiplexed transmission of $109-\mathrm{Tb} / \mathrm{s}$ data signals using homogeneous seven-core fiber," J. Light. Technol., vol. 30, no. 4, pp. 658-665, 2012.

[4] A. Sano, H. Takara, T. Kobayashi, H. Kawakami, H. Kishikawa, T. Nakagawa, Y. Miyamoto, Y. Abe, H. Ono, K. Shikama, M. Nagatani, T. Mori, Y. Sasaki, I. Ishida, K. Takenaga, S. Matsuo, K. Saitoh, M. Koshiba, M. Yamada, H. Masuda, and T. Morioka, "409-Tb/s + 409-Tb/s crosstalk suppressed bidirectional MCF transmission over $450 \mathrm{~km}$ using propagation-direction interleaving," J. Light. Technol., vol. 21, no. 14, pp. 16777-16783, 2013.

[5] J. Sakaguchi, W. Klaus, B. J. Puttnam, J. M. D. Mendinueta, Y. Awaji, N. Wada, Y. Tsuchida, K. Maeda, M. Tadakuma, K. Imamura, R. Sugizaki, T. Kobayashi, Y. Tottori, M. Watanabe, and R. V Jensen, "19-core MCF transmission system using EDFA with shared core pumping coupled via free-space optics.," Opt. Express, vol. 22, no. 1, pp. 90-5, 2014.

[6] S. Matsuo, K. Takenaga, Y. Sasaki, Y. Amma, S. Saito, K. Saitoh, T. Matsui, K. Nakajima, T. Mizuno, H. Takara, Y. Miyamoto, and T. Morioka, "High-Spatial-Multiplicity Multicore Fibers for Future Dense Space-Division-Multiplexing Systems," J. Light. Technol., vol. 34, no. 6, pp. 1464-1475, Mar. 2016.

[7] International Telecommunication Union - ITU-T, "G.694.1 (02/2012), Spectral grids for WDM applications: DWDM frequency grid," Ser. G.694.1, pp. 1-16, 2012.

[8] B. Collings, "New devices enabling software-defined optical networks," IEEE Commun. Mag., vol. 51, no. 3, pp. 66-71, Mar. 2013.

[9] M. M. Filer and S. Tibuleac, "N-degree ROADM Architecture Comparison: Broadcast-and-Select versus Route-and-Select in $120 \mathrm{~Gb} / \mathrm{s}$ DP-QPSK Transmission Systems," in Optical Fiber Communication Conference, 2014, p. Th1I.2.

[10] P. Pavon-Marino, M.-V. Bueno-Delgado, and J.-L. Izquierdo-Zaragoza, "Evaluating Internal Blocking in Noncontentionless Flex-grid ROADMs [Invited]," J. Opt. Commun. Netw., vol. 7, no. 3, p. A474, Mar. 2015.

[11] N. Amaya, M. Irfan, G. Zervas, R. Nejabati, D. Simeonidou, J. Sakaguchi, W. Klaus, B. J. Puttnam, T. Miyazawa, Y. Awaji, N. Wada, and I. Henning, "Fully-elastic multi-granular network with space/frequency/time switching using multi-core fibres and programmable optical nodes," Opt. Express, vol. 21, no. 7, p. 8865, Apr. 2013.

[12] L. E. Nelson, M. D. Feuer, K. Abedin, X. Zhou, T. F. Taunay, J. M. Fini, B. Zhu, R. Isaac, R. Harel, G. Cohen, and D. M. Marom, "Spatial superchannel routing in a two-span ROADM system for space division multiplexing," J. Light. Technol., vol. 32, no. 4, pp. 783-789, 2014.

[13] A. Muhammad, G. Zervas, and R. Forchheimer, "Resource Allocation for Space-Division Multiplexing: Optical White Box Versus Optical Black Box Networking," J. Light. Technol., vol. 33, no. 23, Dec. 2015.

[14] J. Perelló, J. M. Gené, A. Pagès, J. A. Lazaro, and S. Spadaro, "FlexGrid/SDM Backbone Network Design with Inter-Core XT-Limited Transmission Reach," J. Opt. Commun. Netw., vol. 8, no. 8, p. 540, Aug. 2016.

[15] P. Poggiolini, G. Bosco, A. Carena, V. Curri, Y. Jiang, and F. Forghieri, "The GN-Model of Fiber Non-Linear Propagation and its Applications," J. Light. Technol., vol. 32, no. 4, pp. 694-721, Feb. 2014.

[16] A. Carena, G. Bosco, V. Curri, Y. Jiang, P. Poggiolini, and F. Forghieri, "EGN model of non-linear fiber propagation," Opt. Express, vol. 22, no. 13, p. 16335, Jun. 2014.

[17] P. J. Winzer, A. H. Gnauck, A. Konczykowska, F. Jorge, and J.-Y Dupuy, "Penalties from in-band crosstalk for advanced optical modulation formats," 2011 37th Eur. Conf. Exhib. Opt. Commun., no. 1 , pp. 1-3, 2011.

[18] B. J. Puttnam, R. S. Luis, T. A. Eriksson, W. Klaus, J.-M. Delgado Mendinueta, Y. Awaji, and N. Wada, "Impact of Intercore Crosstalk on the Transmission Distance of QAM Formats in Multicore Fibers," IEEE Photonics J., vol. 8, no. 2, pp. 1-9, Apr. 2016.

[19] M. Ruiz, L. Velasco, A. Lord, D. Fonseca, M. Pioro, R. Wessaly, J.P Fernandez-Palacios "Planning Fixed to Flexgrid Gradual Migration: Drivers and Open Issues," IEEE Commun. Mag. pp. 70-76, Jan. 2014.

[20] Lumentum New ROADM Whitebox Preliminary Datasheet [Online]. Available:

http://www.bktel.com/index_htm_files/ROADM\%20Whitebox\%20Targ et\%20Specs.pdf [Last accessed: Mar. 2017].

[21] P.Pavon-Marino and J.-L. Izquierdo-Zaragoza, "Net2Plan: An opensource optical network planning tool for brigding the gap between academia and industry," IEEE Net. Mag., vol. 29, no. 5, pp. 90-96, Oct. 2015.

[22] Net2Plan - The open-source network planner [Online]. Available: http://www.net2plan.com [Last accessed: Jan. 2017].

[23] JOM - The Java Optimization Modeler [Online]. Available: http://www.net2plan.com/jom [Last accessed: Jan. 2017]. 